\title{
Feasibility of Postoperative Spine Stereotactic Body Radiation Therapy in Proximity of Carbon and Titanium Hybrid Implants Using a Robotic Radiotherapy Device
}

\section{Dominik Henzen}

Inselspital Universitatsspital Bern Universitatsklinik fur Radio-Onkologie

Daniel Schmidhalter

Inselspital Universitatsspital Bern Universitatsklinik fur Radio-Onkologie

Gian Guyer

Inselspital Universitatsspital Bern Universitatsklinik fur Radio-Onkologie

Anna Stenger-Weisser

Inselspital Universitatsspital Bern Universitatsklinik fur Radio-Onkologie

Ekin Ermiş

Inselspital Universitatsspital Bern Universitatsklinik fur Radio-Onkologie

Robert Poel

Inselspital Universitatsspital Bern Universitatsklinik fur Radio-Onkologie

Moritz Caspar Deml

Department of orthopedic surgery and traumatology, Inselspital, Bern University Hospital

\section{Michael Karl Fix}

Inselspital Universitatsspital Bern Universitatsklinik fur Radio-Onkologie

\section{Peter Manser}

Inselspital Universitatsspital Bern Universitatsklinik fur Radio-Onkologie

\section{Daniel Matthias Aebersold}

Inselspital Universitatsspital Bern Universitatsklinik fur Radio-Onkologie

Hossein Hemmatazad ( $\nabla$ hossein.hemmatazad@insel.ch )

Inselspital Universitatsspital Bern Universitatsklinik fur Radio-Onkologie https://orcid.org/0000-00034426-6188

\section{Research Article}

Keywords: Postoperative SBRT, Spine metastasis, CFP-T implants, Cyberknife, Dosimetry Analysis

Posted Date: February 1st, 2022 
DOI: https://doi.org/10.21203/rs.3.rs-1303559/v1

License: (c) (1) This work is licensed under a Creative Commons Attribution 4.0 International License. Read Full License 


\section{Abstract}

\section{Background and purpose}

To assess the feasibility of postoperative stereotactic body radiation therapy (SBRT) for patients with hybrid implants consisting of carbon fiber reinforced polyetheretherketone and titanium (CFP-T) using CyberKnife.

\section{Materials and methods}

All essential steps within a radiation therapy (RT) workflow were evaluated. First, the contouring process of target volumes and organs at risk (OAR) was done for patients with CFP-T implants. Second, after RTplanning, the accuracy of the calculated dose distributions was tested in an academic, anthropomorphic phantom using film dosimetry. As a third step, the accuracy of the mandatory image guided radiation therapy (IGRT) including automatic matching was assessed using the anthropomorphic phantom. For this goal, a standard quality assurance (QA) test was modified to carry out its IGRT part in presence of CFP-T implants.

\section{Results}

Using CFP-T implants, target volumes could precisely delineated. There was no need for compromising the contours to overcome artifact obstacles. Differences between measured and calculated dose values were below $11 \%$ for the academic phantom, and at least $95 \%$ of the voxels were within $5 \%$ dose difference. The comparisons for the anthropomorphic phantom showed a gamma-passing rate (5\%, $1 \mathrm{~mm}$ ) of at least $97 \%$. Additionally the test results with and without CFP-T implants were comparable. No issues concerning the IGRT were detected. The modified machine QA test resulted in a targeting error of $0.71 \mathrm{~mm}$, which corresponds to the results of the unmodified standard tests.

\section{Conclusion}

Dose calculation and delivery of postoperative spine SBRT is feasible in proximity of CFP-T implants using a CyberKnife system.

\section{Introduction}

Bone metastases are common in cancer patients and spinal column is involved in approximately two third of osseous metastases. ${ }^{1}$ The treatment options for spine metastases are surgery, radiation therapy (RT), systemic therapy or a multimodality approach using a combination of these therapies. Spine metastases could cause mild to severe pain, pathologic fractures and neurological deficits limiting daily functions and deteriorating the performance status. In case of spinal instability, malignant epidural spinal cord compression (MESCC) with or without neurological symptoms and pathologic vertebral compression fracture (VCF), surgery is the standard of care followed by adjuvant conventional radiation therapy ${ }^{2}$. As for de novo spinal metastases ${ }^{3-5}$, the oncological outcomes are not satisfactory after 
postoperative conventional RT (cRT), in particular the rate of local control (LC) remains low by $30-40 \%$ at 1 year. ${ }^{6-8}$ Therefore, the number of patients who need further treatments after $\mathrm{CRT}$ is notable as they develop recurrent pain or tumor progression. As there is a rapid rise in development and implication of new systemic treatments such as targeted therapies and immunotherapy in recent years, the proportion of cancer survivors is increasing with the urgent need for more effective local treatments to improve the health related quality of life (HRQOL) $)^{9}$. On the other hand, advanced diagnostic imaging modalities are nowadays increasingly available, thus the metastatic sites are better recognized for local treatment as well as in follow-up and re-treatment in case of tumor/symptom progression. ${ }^{10}$ Considering extended life expectancy for patients with malignancies in recent years and with the focus on RT, stereotactic body radiation therapy (SBRT) has become a substantial part of cancer treatment, especially in oligometastatic settings. ${ }^{11}$ Several retrospective studies as well as some prospective data show outstanding local control after SBRT for intact spinal metastases ${ }^{12-15}$. Fewer publications reported excellent oncological outcomes in postoperative setting using SBRT for spinal lesions $s^{2,16,17}$.

The widely used metal implants, mostly made of titanium, offer a reliable stability after spinal surgical interventions ${ }^{18}$. However, they are associated with several drawbacks regarding subsequent RT. The pure titanium implants make notable artifacts on computer tomography (CT) images, which are used to generate RT plans. These artifacts may lead to uncertainties in delineation of target volumes and organs at risk (OAR). For the RT treatment planning, the high density regions could cause inaccuracies in dose calculation ${ }^{19}$. Furthermore, for image guided radiation therapy (IGRT), high quality image information is a key requirement for accurate patient positioning.

Besides titanium implants, the usage of carbon-fiber-composites in orthopedic implants goes back to past decades ${ }^{20,21}$. In addition to their excellent biological and mechanical characteristics, CFP implants reduce artifacts on radiological images and decrease ionizing radiation absorption compared to pure titanium implants, thus support accurate RT planning and delivery ${ }^{22}$. Furthermore, these implants simplify the radiological assessment in the postoperative setting and help the clinicians for a better recognition and treatment of local failures. Considering the fact that nowadays cancer patients have better survival than before, local failures should be diagnosed in time, as patients could profit from an adjuvant treatment. Therefore, there is an increasing clinical interest to use orthopedic implants, which are at least partly made of radiolucent CFP to support the whole RT process and do a better follow up for such patients with spinal metastases.

The current study assesses the RT planning and delivery feasibility of postoperative SBRT in proximity of hybrid implants consisting of titanium and CFP (CFP-T) using a Cyberknife M6 system (CK) (Accuray, Sunnyvale, CA, US).

\section{Materials And Methods}

Essential steps within a RT course were analyzed for phantoms and patients with CFP-T implants. 


\section{Phantom and patient data}

An academic phantom was created by molding a CFP-T implant (Icotec AG, Altstätten, Switzerland) and numerous fiducial markers into epoxy resin (Figure 1 left). The resulting slab can be added to the commercially available RW3 solid water phantom (PTW, Freiburg, Germany). This offers the flexibility to change the depth of the implant as well as carrying out measurements at multiple distally located planes. For this work the CFP-T implant was positioned at a depth $4.5 \mathrm{~cm}$ and measurements were enabled at 0 $\mathrm{mm}, 2 \mathrm{~mm}, 10 \mathrm{~mm}$ and $50 \mathrm{~mm}$ distance distal to the slab with the CFP-T implant in it.

A more realistic and complex situation is represented by an anthropomorphic torso phantom with interchangeable spine inserts (Icotec AG, Altstätten, Switzerland and CIRS, Norfolk, USA) ${ }^{23}$ (Figure 1 right). In the present study, the spine inserts, representing normal bone structure and CFP-T implants were employed. This phantom consists of multiple coronal slabs, which allows carrying out measurements at different planes. Measurements were performed at two different planes in proximity to the CFP-T implant. These planes are indicated and labelled in Figure 2.

Furthermore, planning-CT data sets were available at our institution for two patients with CFP-T implants, who were irradiated postoperatively with cRT and had both pre- and post-operative MRI examinations.

\section{CT and contouring}

CT data sets were acquired for all phantoms as well as for real patients using a Brilliance Big Bore device (Philips, Netherland). Slice thickness was set to 1 and $3 \mathrm{~mm}$ for phantoms and patients, respectively.

All artifacts as well as the high-density titanium parts were contoured and the following densities were assigned to the corresponding regions: $1 \mathrm{~g} / \mathrm{cm}^{3}$ for normal soft tissue, $1.29 \mathrm{~g} / \mathrm{cm}^{3}$ for bone and 4.45 $\mathrm{g} / \mathrm{cm}^{3}$ for titanium.

For the anthropomorphic phantom, two clinically realistic planning target volumes (PTVs) were delineated with consideration of preoperative tumor infiltration, namely PTV1 and PTV2. While the PTV1 encompasses the vertebral body, pedicles and both transverse processes, PTV2 is only confined to vertebral body, unilateral pedicle and left transverse process (Figure 2). In addition, the spinal cord was contoured as OAR.

The planning-CTs, pre- and post-operative spinal MRIs from two patient cases were imported into the treatment planning software Precision (Accuray, Sunnyvale, US) and fused together. The delineation of target volumes on the planning-CTs of patients was done according to consensus contouring guidelines for post-operative SBRT ${ }^{24}$. Briefly, the clinical target volume (CTV) includes the gross residual tumor on postoperative imaging modalities, adjacent anatomical components of the vertebra that are at risk of microscopically spread, areas with preoperative tumor involvement and finally stabilizing implants if the risk of involvement is high ${ }^{24}$. The adjacent relevant OARs are contoured and spinal cord planning risk 
volume (PRV) was generated with $2 \mathrm{~mm}$ expansion. PTV was created adding $2 \mathrm{~mm}$ margin to CTV in all directions and cropped from spinal cord PRV in order to respect the dose constraints.

\section{Treatment planning and dosimetry}

To assess the dosimetric accuracy of the treatment planning system, it is essential to verify that the calculated dose corresponds to the actually delivered dose. For dose delivery, the CK system employs three different beam collimator types, namely fixed collimators, Iris collimator and a multileaf collimator (MLC). In this work the Monte Carlo (MC) dose calculation algorithm, which is available for all collimator types, is used. Statistical uncertainties were set at $1 \%$ for all calculations. For dosimetric comparisons, plans with different collimator types are considered. Due to the two-dimensional (2D) measurement with a high spatial resolution and in accordance with the measurement possibilities within the utilized phantoms, film dosimetry using Gafchromic EBT3 films (Ashland, US) was chosen as dosimetry tool.

Plans with a single perpendicular beam impinging on the academic phantom were created for the Iris collimator and the MLC separately. For both collimators the maximal field size were used. While the $115 \times 100-\mathrm{mm} 2$ field size of the MLC allows covering the whole CFP-T implant, the $60 \mathrm{~mm}$ diameter Iris collimated field was centered on the densest part of the screw.

For the anthropomorphic phantom with inserts, representing CFP-T implants, treatment plans were generated using the Iris collimator and the MLC collimator for both PTVs separately. The four plans were applied and for each delivery, film measurements were carried out at the indicated positions (Figure 2 in Section A).

The same set of four plans was also applied on the anthropomorphic phantom without using the CFP-T implants and the same measurements were carried out as described above.

All irradiated films were digitized using an 10000XL (Epson, JP) scanner, corrected for lateral response artifacts of the scanner ${ }^{25}$ and compared with the calculated dose distributions within the software FilmQA Pro (Ashland, US). In order to convert the grey values on the film into dose values, calibration stripes with a known applied dose were used to carry out a triple channel calibration ${ }^{25}$ within FilmQA Pro. The subsequent comparisons were carried out using the green color channel.

A gamma evaluation with a dose difference criterion of $5 \%$ of the global maximal dose, a distance to agreement criterion of $1 \mathrm{~mm}$ and a $20 \%$ dose threshold was carried out in order to compare the calculated with the measured dose distribution.

In order to better visualize the differences between calculated and measured dose, the dose values for the calibrated films were exported from FilmQA Pro and compared to the calculated dose values using python $3.6^{26}$.

\section{IGRT and delivery}


The CK employs a matching algorithm that matches an orthogonal $\mathrm{kV}$ image pair (actual position of the patient/phantom) to the reference planning CT (2D-3D match) resulting in a correction for the setup error in 6D (translational and rotational setup errors). Inter- and intra-fraction matching is done automatically; this procedure puts a high demand on high quality CT and planar images.

Although, accurate patient/phantom positioning is per se a requirement for the comparisons in the precedent section, a dedicated scenario was created in order to check if the accuracy of the IGRT controlled delivery of the CK can be maintained in presence of CFP-T implants:

Spherically shaped dose distributions are delivered to a cube containing two orthogonal gafchromic films as part of the standard machine QA of the CK. On both films, the deviation of the circular isodose lines from the intended positions are registered as targeting error using the E2E-software (Accuray, Sunnyvale, US). This cube is now affixed to the anthropomorphic phantom and a plan, delivering a spherical dose distribution on the cube is created. However, for patient positioning the spine-tracking matching was used in the area of the phantom where the hybrid implants are positioned. The resulting targeting error was then compared to the results of the regular standard machine QA.

\section{Results}

\section{CT and contouring}

Despite artifacts from CFP-T implants, the spinal cord could be precisely visualized on T2-weighted MRI sequences and contoured. In comparison to SBRT for de novo spinal metastases, there was no need for additional MRI sequences or extra PTV margins. Thus, the influence of artefacts due to the CFP-T implant was not critical.

\section{Treatment planning and dosimetry}

\section{Academic phantom}

Over all comparisons between the measured and calculated dose distributions, the maximal dose differences was lower than $11 \%$ for both collimators. Furthermore, dose differences were less than $5 \%$ of the dose maximum for $95 \%$ of the evaluated voxels, reaching $99 \%$ of the voxels with a dose difference less than $5 \%$ of the dose maximum for the plane in $50 \mathrm{~mm}$ depth. The dose difference plots in Figure 3 show, that the largest differences occur distal to the high-density parts of the screws and in the penumbra region. How well the penumbras match is shown with the isodose lines in Figure $3 e$ ) and f). The measured and calculated dose profiles in Figure 4 show, that the largest differences are present in the plane directly behind the screw, where the calculated dose values are higher than the measured ones. However, for the further distal planes, the calculation seems to underestimate the dose behind the screw.

\section{Anthropomorphic phantom}

The gamma passing rates ( $5 \%$ dose maximum, $1 \mathrm{~mm}$ distance to agreement, $20 \%$ dose threshold) of the comparisons between the measured and calculated dose distributions are summarized in Table 1. A 
passing rate above $90 \%$ is considered as acceptable in clinical routine at our institution, where the measurement and calculations are carried out in a homogenous phantom. This passing rate is well achieved for all comparisons, even within the challenging heterogeneous situation.

For the anthropomorphic phantom equipped with the CFP-T implant, the calculated and measured dose distributions in the Plane 2 are visualized in Figure 5 and 6. Only few regions surpass a dose difference of $5 \%$, while the maximum dose difference is below $11 \%$ of the dose maximum. The isodose line comparison as well as the plotted profiles through the high dose region show an excellent agreement between measurements and calculations.

\section{IGRT and delivery}

The acquired images within the treatment delivery procedure are shown in Figure 7. The digital reconstructed radiograph (DRR) as well as the orthogonal $\mathrm{kV}$ images do not show much deterioration. The quantitative evaluation of the IGRT process, as described in the Materials and Methods section leads to the following results: The targeting error was $0.71 \mathrm{~mm}$, which is in accordance to the achieved accuracy in regular machine QA and well below the actual threshold for those tests $(0.95 \mathrm{~mm})$ as recommended by S. Dieterich et al. ${ }^{27}$.

\section{Discussion}

In the present study, we demonstrate the feasibility of spine SBRT in proximity of CFP-T hybrid implants using Cyberknife.

Treatment of spinal metastases is challenging, using mostly surgery and RT. A randomized study challenged the role of laminectomy for MESCC as surgery showed no benefit in combination with postoperative RT versus RT alone ${ }^{28}$. With further developments of surgical techniques, Patchell et al. showed the superiority of decompressive surgery plus adjuvant RT to RT alone for MESCC in a randomized trial ${ }^{29}$. Besides MESCC, spinal instability is a routine indication for surgical management of metastatic spine. The spine instability neoplastic score (SINS-Score) is a comprehensive classification system and helps the clinicians to select patients who might benefit from a surgical stabilization ${ }^{30}$.

Despite multimodal therapy, the local control and pain response using postoperative conventional RT is not promising. Redmond et al. summarized 12 studies reporting postoperative SBRT for spinal metastases and showed that despite various clinical indications and surgical approaches, patients achieved excellent local control, durable pain response and satisfactory performance status ${ }^{2}$. The safety and efficacy of postoperative spinal SBRT was reported as well in another review article ${ }^{31}$. Until now, only one prospective phase II trial reported the results for postoperative spinal SBRT ${ }^{16}$. With a median followup of 10.5 months, 33 treated patients achieved radiographic and symptomatic excellent local control of $90 \%$ at 1 year with low toxicity profile. Here, the authors mentioned the occasional difficulties regarding 
imaging artifacts caused by spinal metal implants, which made the use of CT-myelogram inevitable to visualize spinal cord.

Postoperative, SBRT has been delivered to tumor sites with metal hardware, traditionally made of titanium $^{18}$. The metal implants are high-Z materials and although offer good stability and stiffness, they can affect the dose distribution to target and normal tissues due to the scattering effects of ionizing radiation ${ }^{32}$. Furthermore, hardware failure is a relevant issue after spine surgery, which could be partly due to irradiation ${ }^{33}$. According to consensus guidelines for postoperative SBRT for spinal metastases, there is no need to include surgical instruments and incision in the CTV ${ }^{34}$. Therefore, someone can hypothesize that SBRT might reduce the rate of hardware failure compared to conventional RT. Unfortunately, there are sparse data regarding type of implants and hardware failure in studies with postoperative spine SBRT.

To overcome those problems with metal hardware, CFP implants have been developed and widely used in spine surgery ${ }^{35}$. CFP cages are biocompatible, promote bone fusion and have been used in surgical interventions since many years ago ${ }^{36,37}$. With further developments, spinal fixation system (rods and screws) was introduced, totally or partially made of CFP. The biomechanical studies showed even benefits of CFP implants in terms of reducing risk of adjacent segment disease (ASD) and hardware failure when compared to titanium fixation system ${ }^{38}$. Considering the stiffness and resistance to motion, CFP implants are equal to titanium and better than pure polyetheretherketone (PEEK) ${ }^{39}$.

Tedesco et al. reported the results of 22 patients with primary spine tumors, who underwent surgical interventions using composite CFP fixation system ${ }^{40}$. Unfortunately, there are no details about the type of RT and authors concluded that CFP implants are comparable to titanium regarding complications, stability at weight bearing and functional recovery ${ }^{40}$. In another study from the same group, a mixed cohort including 20 primary and 14 metastatic spine tumor patients were treated with CFP composite implants ${ }^{41}$. All cases had postoperative RT, here again there is no reported data about type and intention of RT. With a mean follow up of 13 months (6 - 36 months), only two cases (6\%) had hardware failure due to local recurrence, the overall rate of local failure was $17.6 \%(6 / 34)^{41}$.

In 2017, Ringel et al. published a study consisting of 35 patients, mostly with spinal metastasis, after posterior stabilization using CFP-T pedicle screws (Icotec, Altstätten, Switzerland) ${ }^{42}$. The study evaluated the feasibility of CFP-T fixation system and their impact on postoperative imaging as well as radiotherapy planning. Of interest, RT plans were made for both photons and protons. Matched controls with titanium were used to assess the absorption of ionizing radiation and imaging artifacts in patients with CFP-T implants. Almost all screws (1 revision out of 251) were placed correctly through the pedicles as seen on postoperative imaging. For RT treatment plans, notably smaller CT-Hounsfield values for CFPT implants compared to titanium screws improved the precision of dose calculation, especially for proton beams. The CFP-T screws used in above study are titanium-coated in the pedicle part to improve 
osseointegration and better fusion to the bone, as it was shown in a prospective comparative study between titanium-coated and uncoated PEEK cages in lumbar surgery ${ }^{43}$.

In our study, we assessed the essential steps in RT workflow for postoperative spine SBRT in proximity of CFP-T hybrid implants using a Cyberknife. The fusion of pre- and postoperative MRI-sequences to our planning CT as well as contouring process were carried out successfully. Here, we focused on recommendations from international consensus guidelines for postoperative spine SBRT ${ }^{24}$. As epidural space is the most common site of treatment failure after postoperative SBRT ${ }^{44}$, we contoured the true spinal cord instead of thecal sac to avoid underdosage and improve LC. Furthermore, the achieved accuracy using CFP-T hybrid implants is comparable to SBRT for de novo spinal metastases and there was no need for enlarged CTV to PTV margins. For treatment planning, including dose calculation, a density correction was applied to the high-density parts as well as the artifacts. Technical solutions like dual energy CT, which might reduce the artifacts are not clinical standard at our institution. Thus, the application of such technologies is beyond the scope of this work but will be evaluated in future studies.

The comparison between all calculated and measured situations show a very good agreement. Both, the maximal dose deviations of $11 \%$ as well as the gamma passing rates or occurring dose differences ( $95 \%$ of the voxels $<5 \%$ of dose maximum) build confidence in the accuracy of the dose calculation algorithm. Where possible, the standard situation (no implant) was used as comparison. The results for both situations were equally good.

The IGRT including the automatic matching algorithm did not show any issues throughout all executed tests. Convincing were also the results of the modified machine QA test, which were in the range of the regular standard tests and well within the recommended thresholds ${ }^{27}$.

\section{Conclusion}

Based on the results of this study, we have shown the dosimetric and delivery feasibility of postoperative spine SBRT in proximity of CFP-T implants using a CyberKnife machine.

\section{Abbreviations}

SBRT Stereotactic Body Radiation Therapy

CFP-T Carbon Fiber reinforced Polyetheretherketone and Titanium

RT Radiation Therapy

OAR Organ at Risk

IGRT Image Guided Radiation Therapy

QA Quality Assurance

Page $10 / 22$ 
MESCC Malignant Epidural Spinal Cord Compression

VCF Vertebral Compression Fracture

cRT Conventional RT

LC Local Control

HRQOL Health Related Quality Of Life

CT Computer Tomography

CK CyberKnife

PTV Planning Target Volume

CTV Clinical Target Volume

PRV Planning Risk Volume

MLC Multileaf Collimator

MC Monte Carlo

2D Two Dimensional

DRR Digital Reconstructed Radiograph

SINS Spine Instability Neoplastic Score

ASD Adjacent Segment Disease

PEEK Polyetheretherketone

\section{Declarations}

Ethical Approval and Consent to participate: Not applicable

Consent for publication: Not applicable

Availability of supporting data: Not applicable

Competing interests: None

Funding: None

Authors' contributions: All authors contributed equally for the manuscript 
Acknowledgements: The authors thank Icotec AG for providing us with the anthropomorphic phantom along with the different inserts as well as the CFP-T implant for the academic phantom.

\section{References}

1. Spratt DE, Beeler WH, de Moraes FY, et al. An integrated multidisciplinary algorithm for the management of spinal metastases: an International Spine Oncology Consortium report. Lancet Oncol. 2017;18(12):e720-e730. doi:10.1016/S1470-2045(17)30612-5

2. Redmond KJ, Lo SS, Fisher C, Sahgal A. Postoperative Stereotactic Body Radiation Therapy (SBRT) for Spine Metastases: A Critical Review to Guide Practice. Int J Radiat Oncol Biol Phys. 2016;95(5):1414-1428. doi:10.1016/j.ijrobp.2016.03.027

3. Van Der Linden YM, Lok JJ, Steenland E, et al. Single fraction radiotherapy is efficacious: A further analysis of the Dutch Bone Metastasis Study controlling for the influence of retreatment. Int J Radiat Oncol Biol Phys. 2004;59(2):528-537. doi:10.1016/j.jirobp.2003.10.006

4. Harstell WF, Scott CB, Bruner DW, et al. Randomized trial of short- versus long-course radiotherapy for palliation of painful bone metastases. J Nat/ Cancer Inst. 2005;97(11):798-804. doi:10.1093/jnci/dji139

5. Foro Arnalot P, Fontanals AV, Galcerán JC, et al. Randomized clinical trial with two palliative radiotherapy regimens in painful bone metastases: 30 Gy in 10 fractions compared with $8 \mathrm{~Gy}$ in single fraction. Radiother Oncol. 2008;89(2):150-155. doi:10.1016/j.radonc.2008.05.018

6. Sellin JN, Suki D, Harsh V, et al. Surgery for Spinal Metastases From Thyroid Carcinoma. 2015;23(October):419-428. doi:10.3171/2015.1.SPINE14431.Disclosure

7. Klekamp J, Samii M. Surgical results for spinal meningiomas. Surg Neurol. 1999;52(6):552-562. doi:10.1016/S0090-3019(99)00153-6

8. Epstein-Peterson ZD, Sullivan A, Krishnan M, et al. Postoperative radiation therapy for osseous metastasis: Outcomes and predictors of local failure. Pract Radiat Oncol. 2015;5(5):e531-e536. doi:10.1016/j.prro.2015.02.006

9. Pucci C, Martinelli C, Ciofani G. Innovative approaches for cancer treatment: Current perspectives and new challenges. Ecancermedicalscience. 2019;13:1-26. doi:10.3332/ecancer.2019.961

10. Shinagare AB, Khorasani R. Evidence-based cancer imaging. Korean J Radiol. 2017;18(1):107-112. doi:10.3348/kjr.2017.18.1.107

11. Desai NB, Laine AM, Timmerman RD. Stereotactic ablative body radiotherapy (SAbR) for oligometastatic cancer. Br J Radiol. 2017;90(1070). doi:10.1259/bjr.20160500

12. Amdur RJ, Bennett J, Olivier K, et al. A Prospective, phase II study demonstrating the potential value and limitation of radiosurgery for spine metastases. Am J Clin Oncol Cancer Clin Trials. 2009;32(5):515-520. doi:10.1097/COC.0b013e318194f70f

13. Sprave T, Verma V, Förster R, et al. Randomized phase II trial evaluating pain response in patients with spinal metastases following stereotactic body radiotherapy versus three-dimensional conformal 
radiotherapy. Radiother Oncol. 2018;128(2):274-282. doi:10.1016/j.radonc.2018.04.030

14. Zeng KL, Tseng CL, Soliman H, Weiss Y, Sahgal A, Myrehaug S. Stereotactic body radiotherapy (SBRT) for oligometastatic spine metastases: An overview. Front Oncol. 2019;9(MAY):1-11. doi:10.3389/fonc.2019.00337

15. Husain ZA, Sahgal A, De Salles A, et al. Stereotactic body radiotherapy for de novo spinal metastases: Systematic review International Stereotactic Radiosurgery Society practice guidelines. J Neurosurg Spine. 2017;27(3):295-302. doi:10.3171/2017.1.SPINE16684

16. Redmond KJ, Sciubba D, Khan M, et al. A Phase 2 Study of Post-Operative Stereotactic Body Radiation Therapy (SBRT) for Solid Tumor Spine Metastases. Int J Radiat Oncol Biol Phys. 2020;106(2):261-268. doi:10.1016/j.jijobp.2019.10.011

17. Tao R, Bishop AJ, Brownlee Z, et al. Stereotactic Body Radiation Therapy for Spinal Metastases in the Postoperative Setting: A Secondary Analysis of Mature Phase 1-2 Trials. Int J Radiat Oncol Biol Phys. 2016;95(5):1405-1413. doi:10.1016/j.jjrobp.2016.03.022

18. Grob D, Daehn S, Mannion AF. Titanium mesh cages (TMC) in spine surgery. Eur Spine J. 2005;14(3):211-221. doi:10.1007/s00586-004-0748-7

19. Jäkel O, Reiss P. The influence of metal artefacts on the range of ion beams. Phys Med Biol. 2007;52(3):635-644. doi:10.1088/0031-9155/52/3/007

20. Ciappetta P, Boriani S, Fava GP. A carbon fiber reinforced polymer cage for vertebral body replacement: Technical note. Neurosurgery. 1997;41(5):1203-1206. doi:10.1097/00006123199711000-00040

21. Lipka JM, Ranu HS. The Role of Carbon Fibers in Orthopedic Implants: A Review. In: Materials Sciences and Implant Orthopedic Surgery. Springer Netherlands; 1986:335-343. doi:10.1007/978-94009-4474-9_25

22. Nevelsky A, Borzov E, Daniel S, Bar-Deroma R. Perturbation effects of the carbon fiber-PEEK screws on radiotherapy dose distribution. J Appl Clin Med Phys. 2017;18(2):62-68. doi:10.1002/acm2.12046

23. Poel R, Belosi F, Albertini F, et al. Assessing the advantages of CFR-PEEK over titanium spinal stabilization. Phys Med Biol. Published online 2020:11.

24. Redmond KJ, Robertson S, Lo SS, et al. Consensus Contouring Guidelines for Postoperative Stereotactic Body Radiation Therapy for Metastatic Solid Tumor Malignancies to the Spine. Int J Radiat Oncol Biol Phys. 2017;97(1):64-74. doi:10.1016/j.jirobp.2016.09.014

25. Micke A, Lewis DF, Yu X. Multichannel film dosimetry with nonuniformity correction. 2011;07470(February):2523-2534. doi:10.1118/1.3576105

26. van Rossum G, Drake FL. The Python Language Reference Manual.; 2009.

27. Dieterich S, Cavedon C, Chuang CF, et al. Report of AAPM TG 135: Quality assurance for robotic radiosurgery. Med Phys. 2011;38(6):2914-2936. doi:10.1118/1.3579139

28. Young RF, Post EM, King GA. Treatment of spinal epidural metastases. Randomized prospective comparison of laminectomy and radiotherapy. J Neurosurg. 1980;53(6):741-748. 
doi:10.3171/jns.1980.53.6.0741

29. Patchell RA, Tibbs PA, Regine WF, et al. Direct decompressive surgical resection in the treatment of spinal cord compression caused by metastatic cancer: A randomised trial. Lancet. 2005;366(9486):643-648. doi:10.1016/S0140-6736(05)66954-1

30. Fourney DR, Frangou EM, Ryken TC, et al. Spinal Instability Neoplastic Score: An Analysis of Reliability and Validity From the Spine Oncology Study Group. 2018;29(22). doi:10.1200/JCO.2010.34.3897

31. Alghamdi M, Tseng CL, Myrehaug S, et al. Postoperative stereotactic body radiotherapy for spinal metastases. Chinese Clin Oncol. 2017;6(Suppl 2):1-11. doi:10.21037/cco.2017.06.27

32. Wang X, Yang JN, Li X, et al. Effect of spine hardware on small spinal stereotactic radiosurgery dosimetry. Phys Med Biol. 2013;58(19):6733-6747. doi:10.1088/0031-9155/58/19/6733

33. Pedreira R, Abu-Bonsrah N, Karim Ahmed A, et al. Hardware failure in patients with metastatic cancer to the spine. J Clin Neurosci. 2017;45:166-171. doi:10.1016/j.jocn.2017.05.038

34. Redmond KJ, Lo SS, Soltys SG, et al. international survey. 2018;26(3):299-306. doi:10.3171/2016.8.SPINE16121.Consensus

35. Li CS, Vannabouathong C, Sprague S, Bhandari M. The use of carbon-fiber-reinforced (CFR) peek material in orthopedic implants: A systematic review. Clin Med Insights Arthritis Musculoskelet Disord. 2014;8:33-45. doi:10.4137/CMAMD.S20354

36. Kabir SMR, Alabi J, Rezajooi K, Casey ATH. Anterior cervical corpectomy: Review and comparison of results using titanium mesh cages and carbon fibre reinforced polymer cages. $\mathrm{Br} J$ Neurosurg. 2010;24(5):542-546. doi:10.3109/02688697.2010.503819

37. Kersten RFMR, Van Gaalen SM, De Gast A, Öner FC. Polyetheretherketone (PEEK) cages in cervical applications: A systematic review. Spine J. 2015;15(6):1446-1460. doi:10.1016/j.spinee.2013.08.030

38. Chou WK, Chien A, Wang JL. Biomechanical analysis between PEEK and titanium screw-rods spinal construct subjected to fatigue loading. J Spinal Disord Tech. 2015;28(3):E121-E125. doi:10.1097/BSD.0000000000000176

39. Bruner HJ, Guan Y, Yoganandan N, Pintar FA, Maiman DJ, Slivka MA. Biomechanics of polyaryletherketone rod composites and titanium rods for posterior lumbosacral instrumentation: Presented at the 2010 Joint Spine Section Meeting - Laboratory investigation. J Neurosurg Spine. 2010;13(6):766-772. doi:10.3171/2010.5.SPINE09948

40. Tedesco G, Gasbarrini A, Bandiera S, Ghermandi R, Boriani S. Composite PEEK/Carbon fiber implants can increase the effectiveness of radiotherapy in the management of spine tumors. J Spine Surg. 2017;3(3):323-329. doi:10.21037/jss.2017.06.20

41. Boriani S, Tedesco G, Ming L, et al. Carbon-fiber-reinforced PEEK fixation system in the treatment of spine tumors: a preliminary report. Eur Spine J. 2018;27(4):874-881. doi:10.1007/s00586-017-5258-5

42. Ringel F, Ryang YM, Kirschke JS, et al. Radiolucent Carbon Fiber-Reinforced Pedicle Screws for Treatment of Spinal Tumors: Advantages for Radiation Planning and Follow-Up Imaging. World Neurosurg. 2017;105:294-301. doi:10.1016/j.wneu.2017.04.091 
43. Kashii M, Kitaguchi K, Makino T, Kaito T. Comparison in the same intervertebral space between titanium-coated and uncoated PEEK cages in lumbar interbody fusion surgery. J Orthop Sci. 2019;25(4). doi:10.1016/j.jos.2019.07.004

44. Chan MW, Thibault I, Atenafu EG, et al. Patterns of epidural progression following postoperative spine stereotactic body radiotherapy: implications for clinical target volume definition. 2016;d(April):652-659. doi:10.3171/2015.6.SPINE15294.652

\section{Table}

Table 1: Gamma passing rates of the comparison between measured and calculated dose distributions for the anthropomorphic phantom. The position of the measurement planes is indicated in Figure 2.

\begin{tabular}{|llll|}
\hline & Collimator & Phantom & PTV 1 / PTV 2 \\
\hline Plane 1 & Iris & Bone & $100 \% / 97 \%$ \\
& & CFP-T implant & $100 \% / 97 \%$ \\
& MLC & Bone & $99 \% / 97 \%$ \\
\hline Plane 2 & Iris & CFP-T implant & $100 \% / 99 \%$ \\
& & CFP-T implant & $100 \% / 99 \%$ \\
& MLC & Bone & $99 \% / 97 \%$ \\
\hline & & CFP-T implant & $98 \% / 97 \%$ \\
\hline
\end{tabular}

\section{Figures}




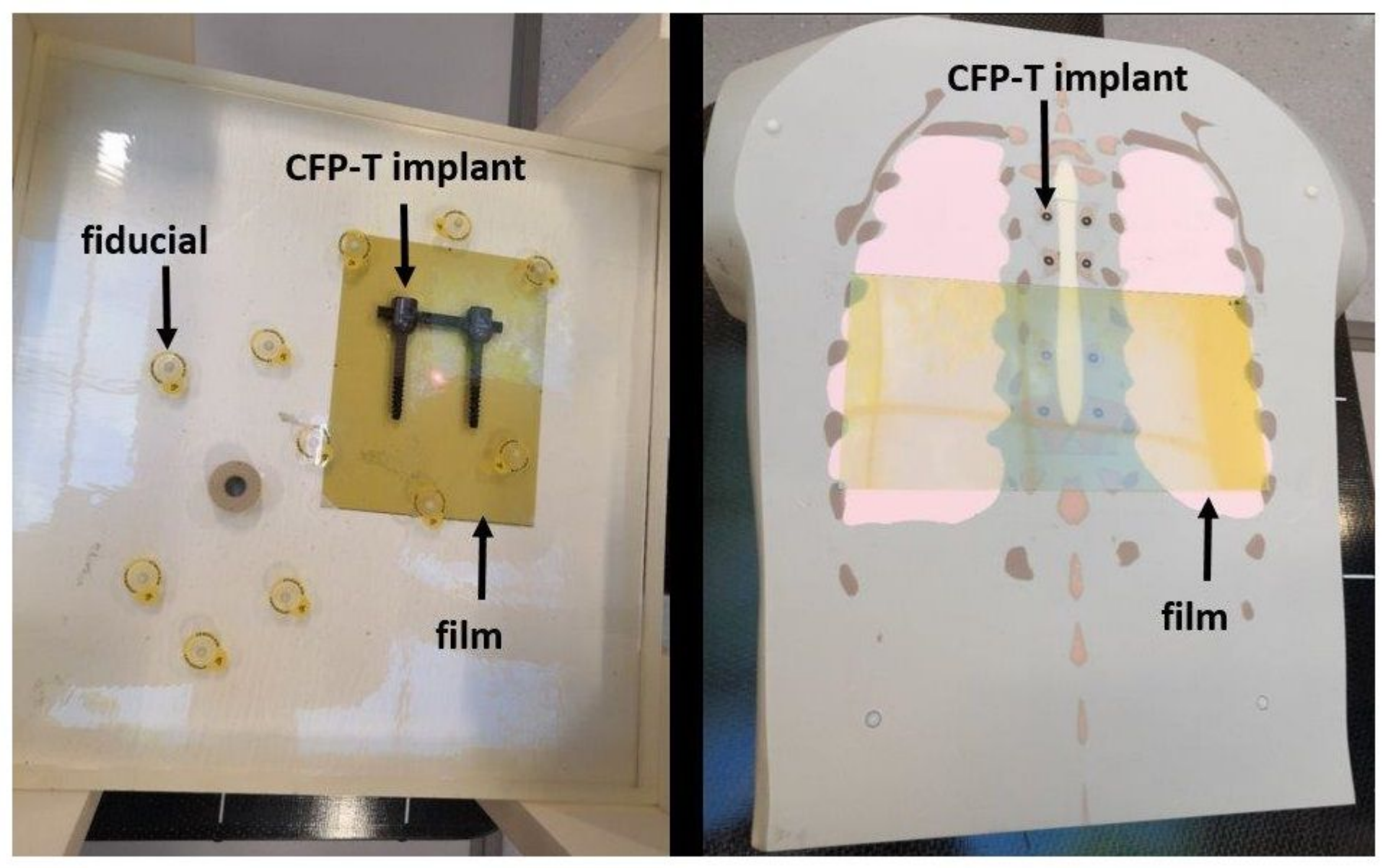

\section{Figure 1}

Image of the academic (left) and the anthropomorphic phantom (right).

The left picture shows molded slab on the solid water phantom. The hybrid CFP-T (black) as well as the fiducials (inside the yellow circles) are visible. On the right side, the disassembled anthropomorphic phantom with the CFP-T implant is shown. The gafchromic films in yellow on both images indicate the area where measurements were carried out. 


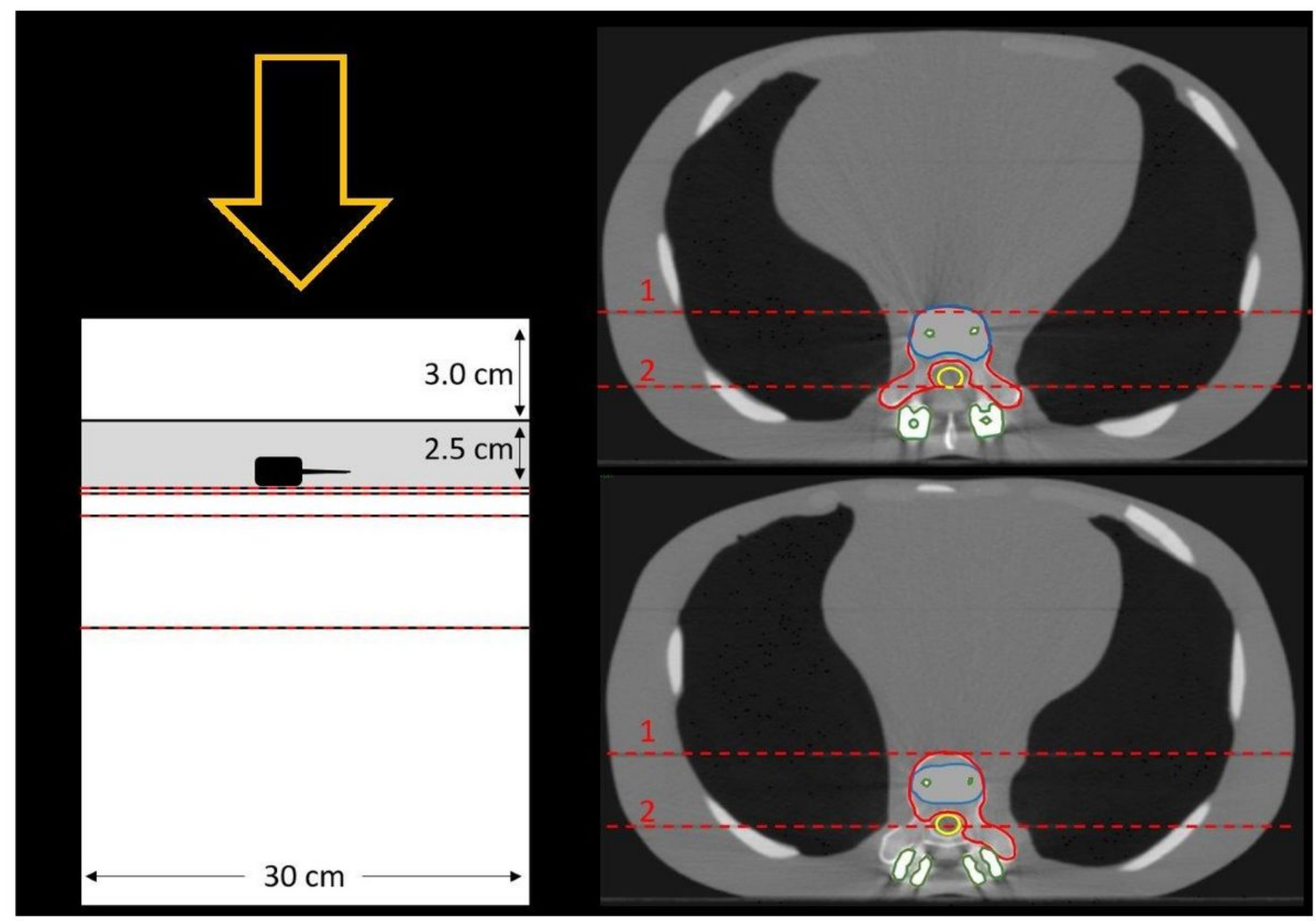

Figure 2

a) A schematic view of the academic phantom with the epoxy slab in grey and the CFP-T implant in black. b,c) The contoured PTVs of the anthropomorphic phantom (red), the spinal cord (yellow), the high density components (green) and bony areas (blue) for PTV1 (b)) and PTV2(c)) respectively. The red dashed lines on all images indicate the planes where film measurements were carried out. 
a)

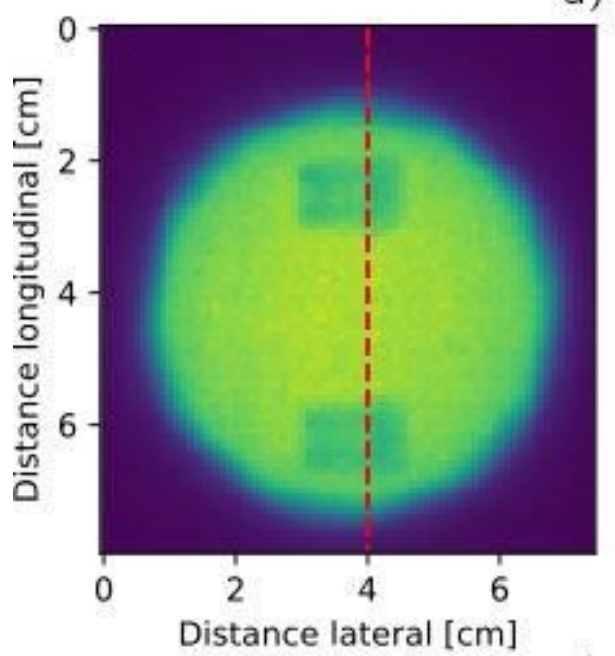

b)

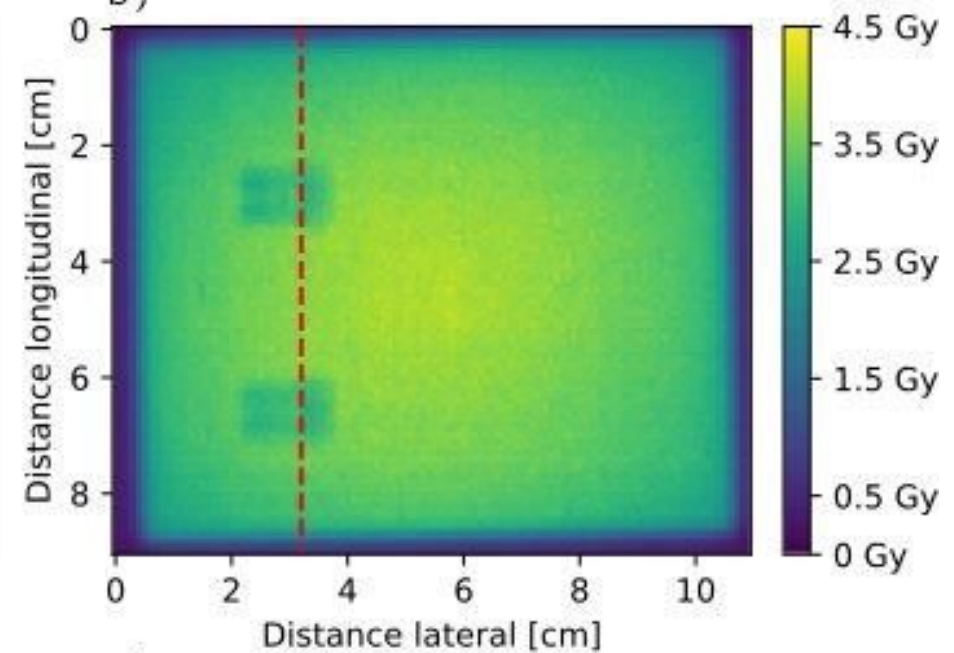

c)

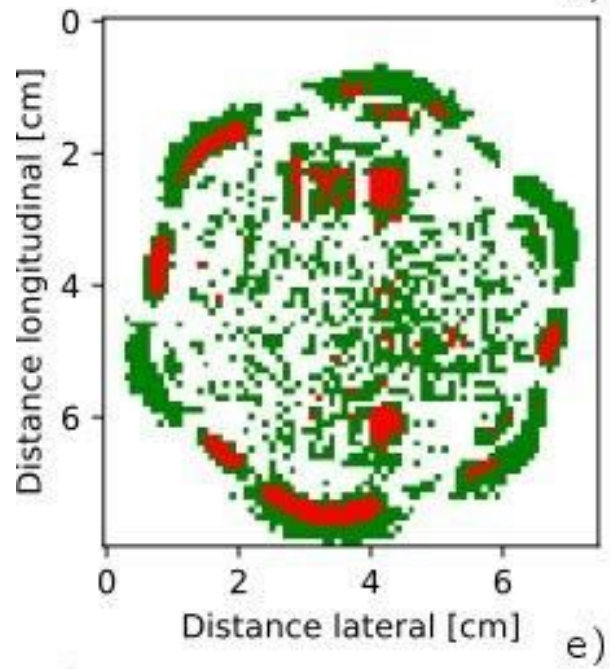

d)
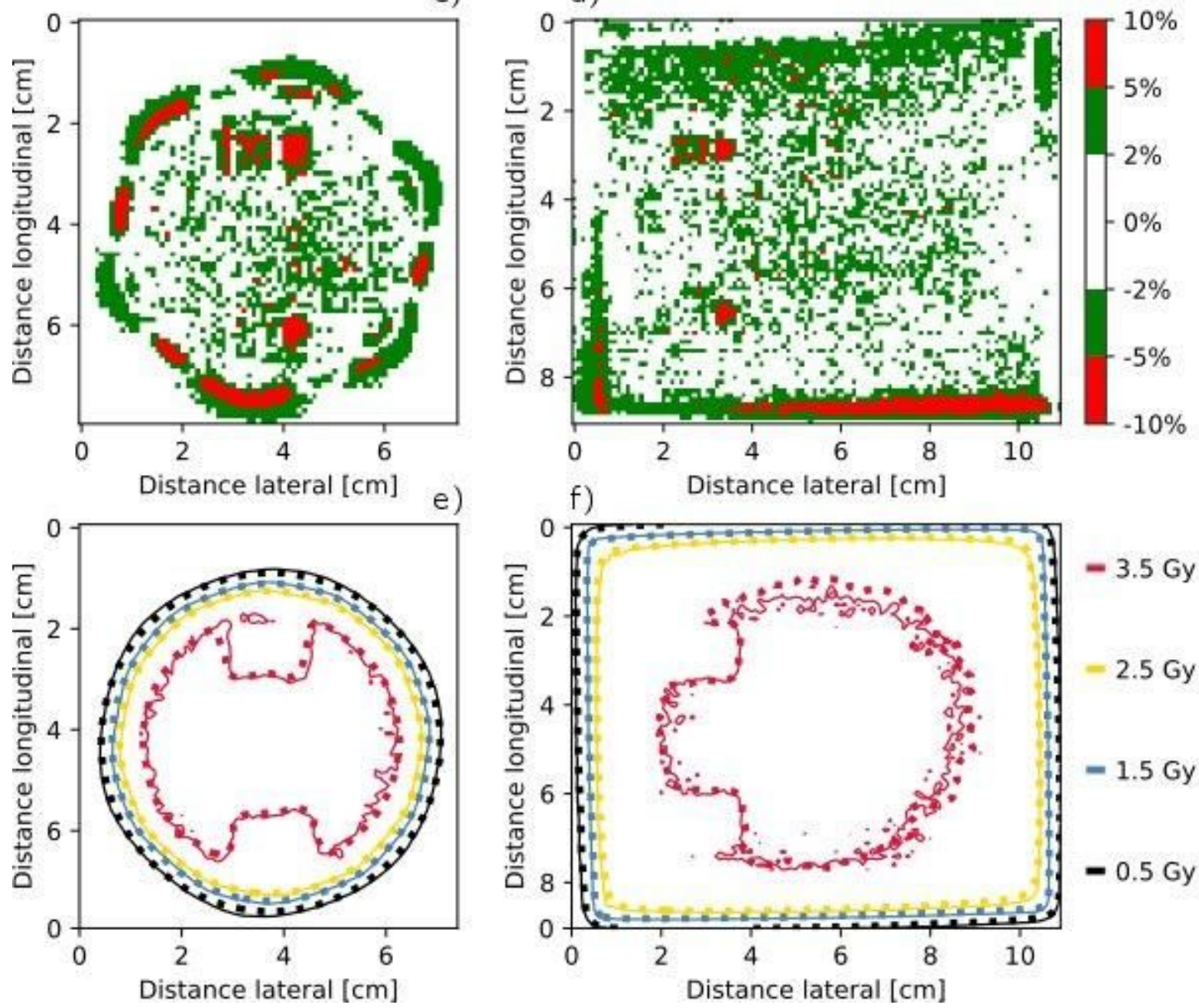

f) Distance lateral $[\mathrm{cm}]$

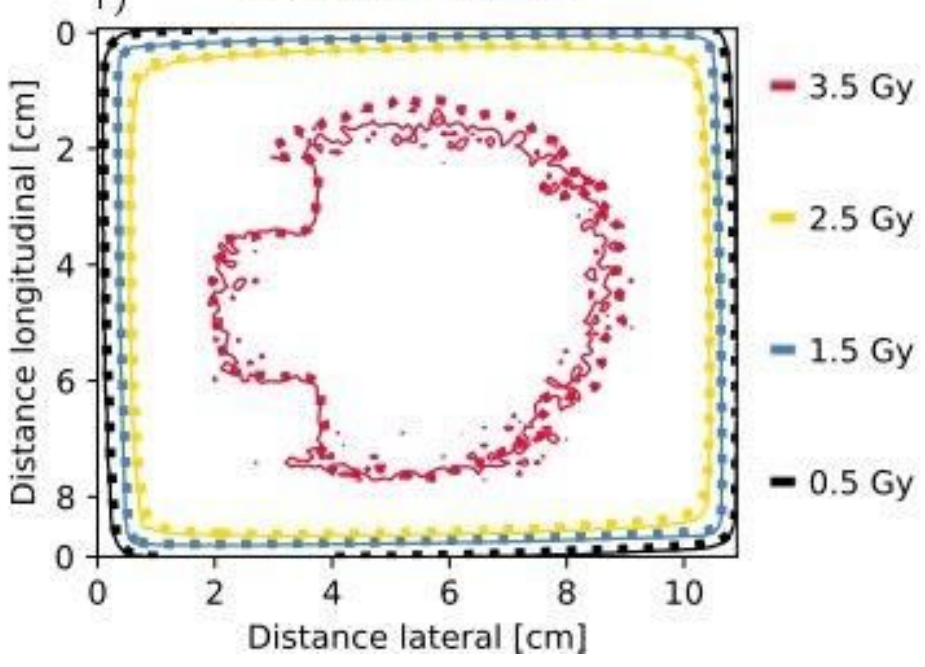

Figure 3

Film measured dose distribution in the measurement plane directly adjacent to the CFP-T implant, as shown in Figure 2a), for the Iris and MLC collimated beam in a) and b), respectively. Dose difference between the measured and calculated dose distribution in c) and d) for the Iris and the MLC collimated beam, respectively. Isodose lines of the measured (solid lines) and calculated (dotted) dose distribution in e) and f) for the Iris and the MLC collimated beam, respectively. 

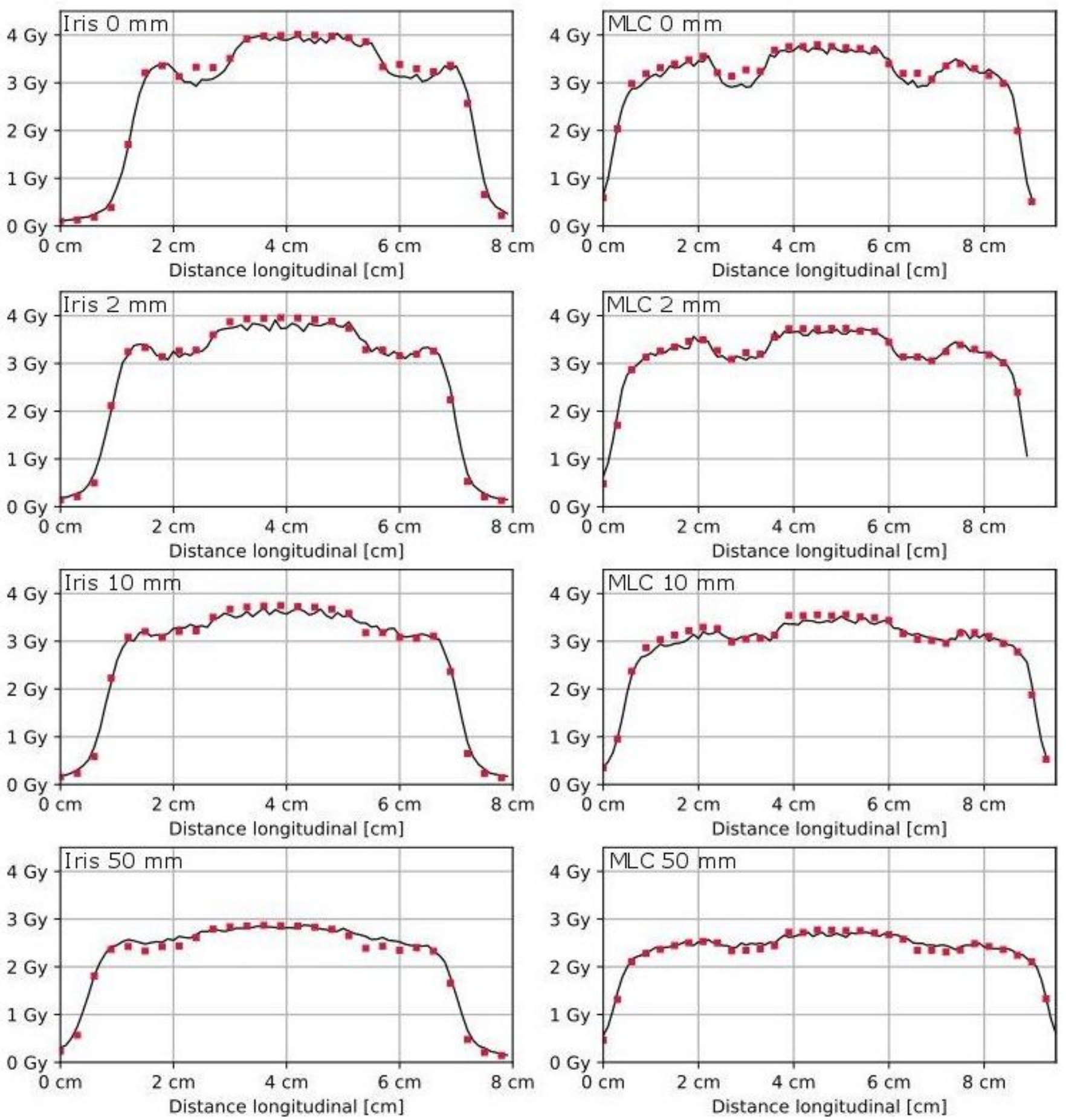

Figure 4

Dose Profiles for all measurement planes $(0,2,10$ and $50 \mathrm{~mm}$ distal to the CFP-T implant) within the academic phantom as indicated in Figure 3 by the red lines (Iris left and MLC right). Distal distance from the CFP-T implant as well as used collimation device are indicated in each subfigure. 
a)

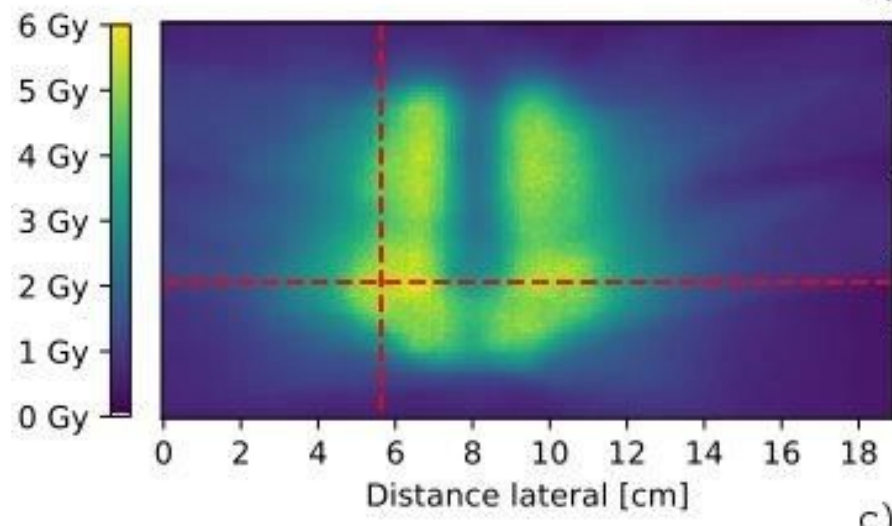

b)

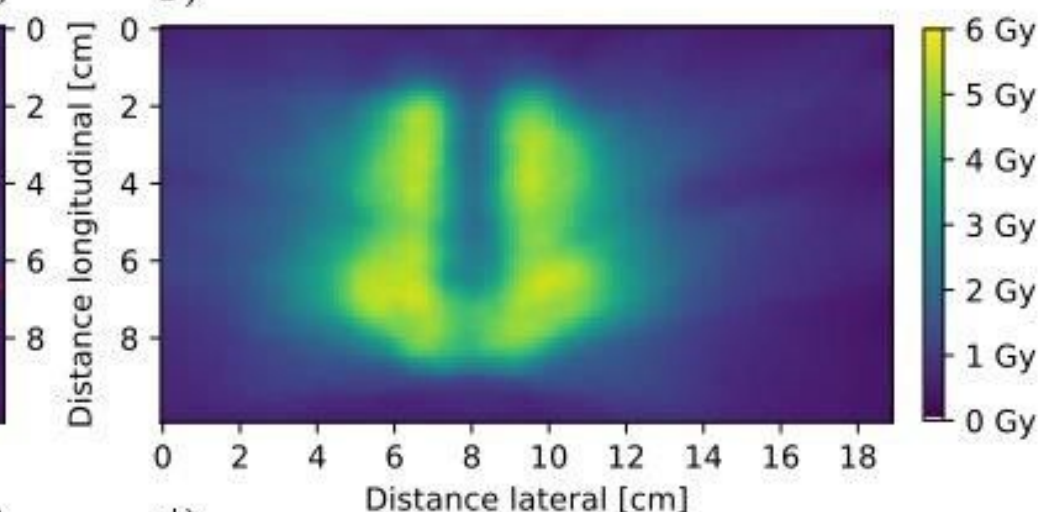

c)

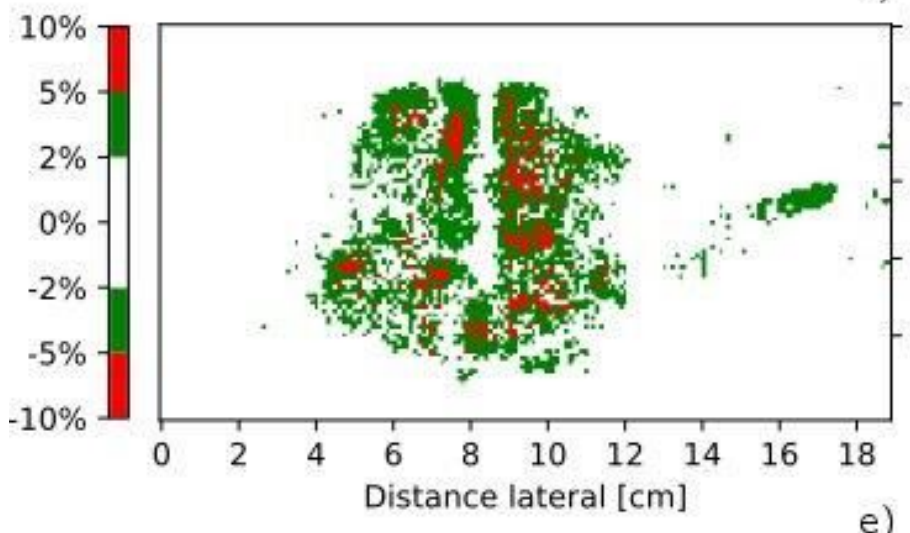

d) Distance lateral $[\mathrm{cm}]$
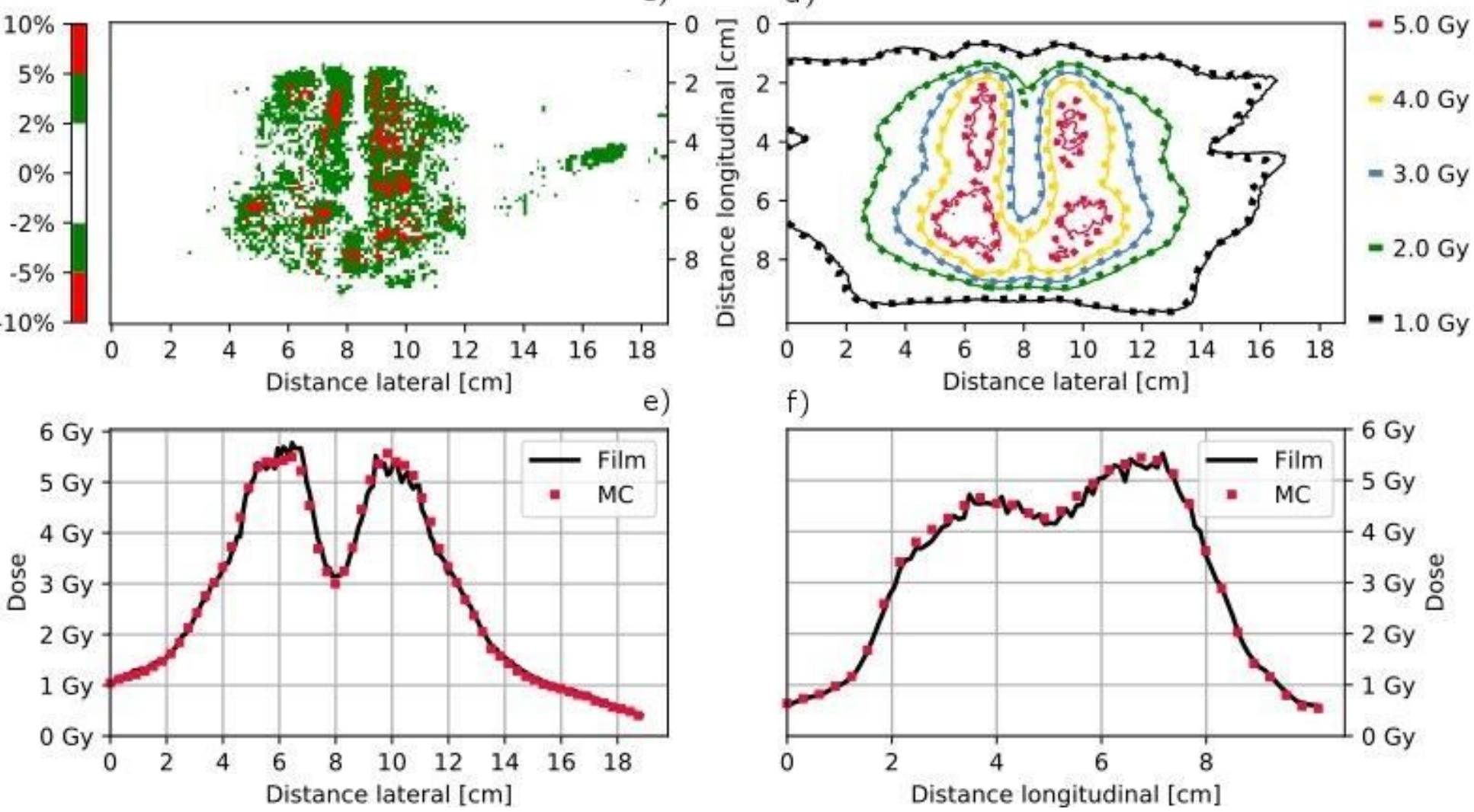

f)

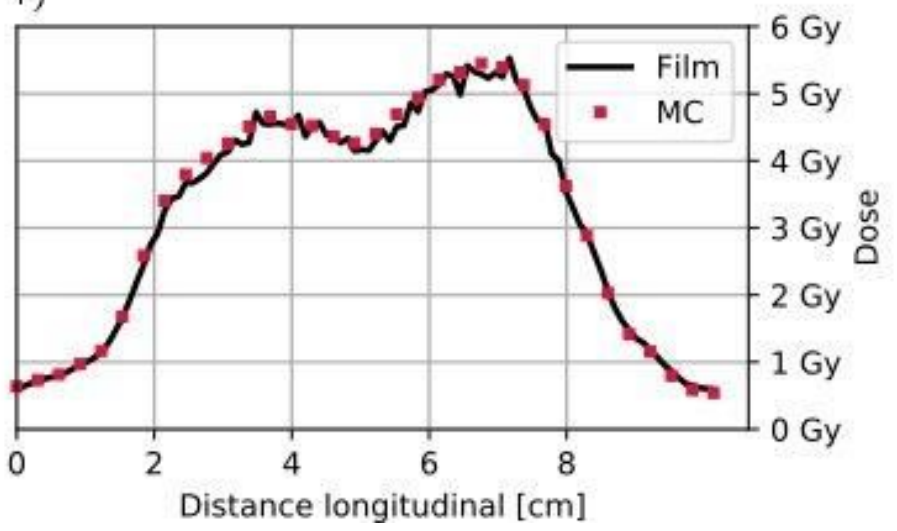

Figure 5

Film measured (a) and MC calculated (b) sagittal dose distribution in Plane 2 for the PTV1 as indicated in Figure $2 \mathrm{~b}$ ) using the MLC collimator and the composite phantom. The dose difference (Film-MC) is shown in $\mathrm{C}$ ) and an overlay of the isodose lines ( $\mathrm{MC}=$ dotted) is shown in $\mathrm{c}$ ) and d), respectively. Horizontal e) and transversal f) dose profiles are indicated in a). 
a)

b)
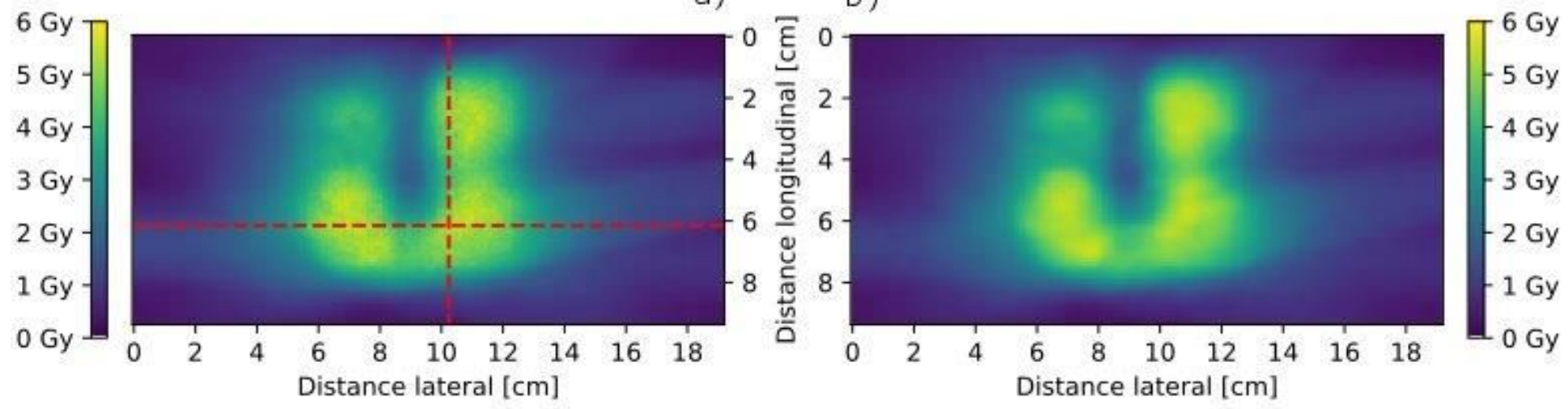

c)

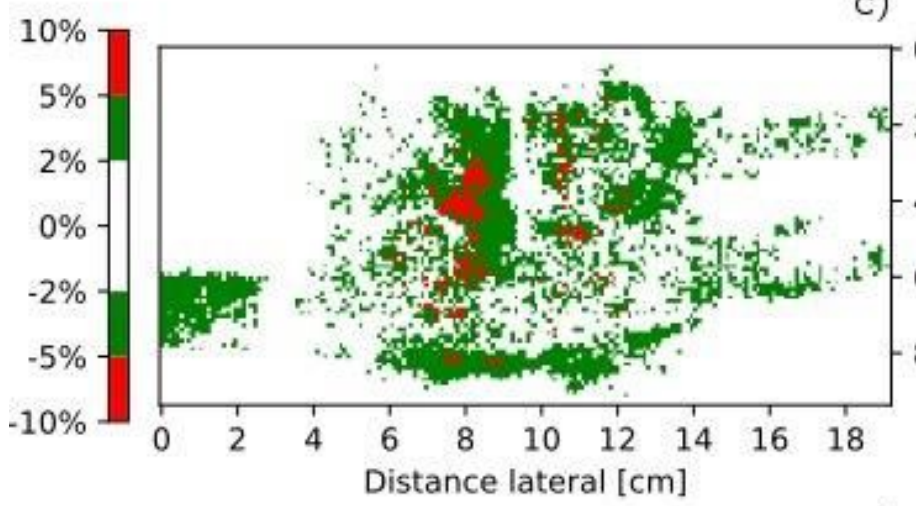

d)

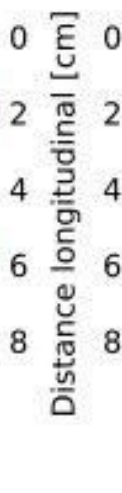

e)
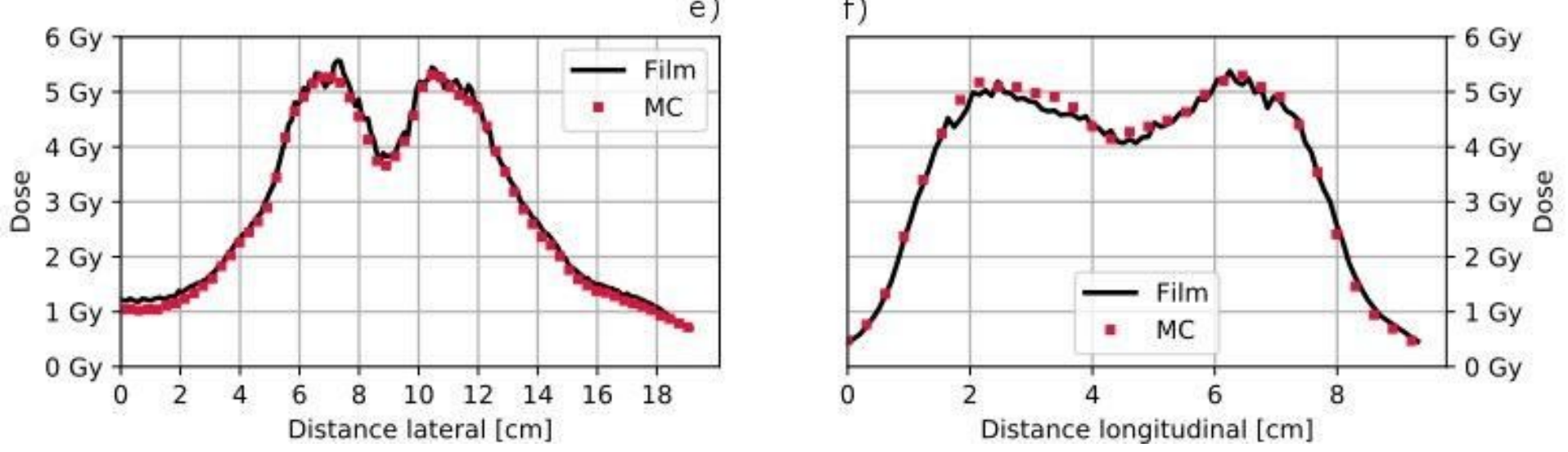

Figure 6

Film measured (a) and MC calculated (b) sagittal dose distribution in Plane 2 for the PTV2 in the posterior plane indicated in Figure $2 \mathrm{c}$ ) using the Iris collimator and the composite phantom. The dose difference (Film-MC) is shown in $\mathrm{c}$ ) and an overlay of the isodose lines (MC = dotted) is shown in $\mathrm{d}$ ). Horizontal e) and transversal f) dose profiles are indicated in a). 


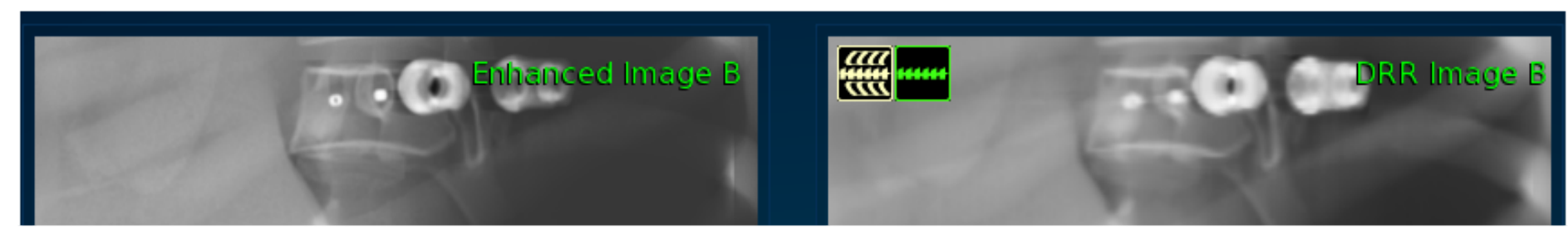

\section{Figure 7}

Live image for both $\mathrm{x}$-ray cameras on a) and c) as well as the corresponding calculated DDR images on b) and d). 